\title{
The Italian Society of Surgical Oncology (SICO) survey on the minimum requirements of rare cancers referral centers
}

\author{
Alessandro Gronchi ${ }^{1} \cdot$ Paolo Delrio $^{2} \cdot$ Vittorio Quagliuolo $^{3} \cdot$ Sergio Sandrucci $^{4}$
}

Received: 15 May 2016/Accepted: 21 May 2016/Published online: 16 June 2016

(c) Italian Society of Surgery (SIC) 2016

With 366,000 new cases and 175,000 deaths per year [1], tumors are the second leading cause of death and morbidity in Italy [1]. Great interest and most resources are directed at the most common cancers, "the big killers" that, even today, involve thousands of people every year. However, there are actually many different and clinically relevant types of neoplasms, the so called "rare cancers", which receive far less attention.

Rare cancers are those with an incidence of less than $6 / 100,000$ per year, corresponding to less than 30,000 new cases per year in Europe. [2] Despite the rarity of each rare cancer, they constitute all together $22 \%$ of all cancers diagnosed in the European Union each year, that is more than half a million new rare cancer cases each year, with 4,300,000 people in Europe currently living with rare cancers. The European project RARECARENet [2], twinned with the Italian project RITA2 [3] has identified, through a consensus process among several experts, about 186 different histotypes. Rare cancers can also arise in common sites, as for instance breast, prostate, lung and many others.

Sergio Sandrucci

sergio.sandrucci@unito.it

1 Department of Cancer Medicine and Surgical Oncology, Fondazione IRCCS Istituto Nazionale dei Tumori, Milan, Italy

2 Department of Surgical Oncology, Istituto Nazionale per lo Studio e la Cura dei Tumori Fondazione Giovanni Pascale IRCCS, Naples, Italy

3 Oncologic Surgery and Sarcoma Unit, Humanitas Cancer Center, Rozzano, Italy

4 Sarcoma Unit, Department of Surgery, Universiy of Turin, Turin, Italy
A recent collaborative study, based on data collected by the network of Italian Cancer Registries (AIRTUM), describes the burden of rare cancers in Italy [4]. They account for $25 \%$ of new oncologic diagnoses in Italy; $7 \%$ are hematological, and $18 \%$ solid neoplasms; 85,000 new cases/year are expected, with about 600,000 people living with a diagnosis of rare cancer.

Among solid rare cancers epithelial tumours of the digestive system are the most common $(23 \%)$, followed by epithelial tumours of head and neck $(17 \%)$, rare cancers of the female genital system (17\%), endocrine tumours (13\% including thyroid carcinomas and less than $1 \%$ excluding thyroid carcinomas), sarcomas (8\%), central nervous system tumours and rare epithelial tumours of the thoracic cavity (5\%). Rare male genital, eye, neuroendocrine, embryonal, skin tumours and malignant melanoma of mucosae constitute $<4 \%$ of all solid rare cancers [4].

The challenges of rare cancers are the limited number of reference centers, both in individual countries and in Europe, with a consequent delayed treatment, diagnostic uncertainty due to the lack of information and limited access to appropriate therapies and/or clinical expertise. To ensure an adequate care and management is necessary to rethink the way research and care are organized, in order to make knowledge migrate instead of patients. One of the recommendations of the "Call to action against rare cancers" of Rare Cancers Europe, a multistakeholder initiative dedicated to putting firmly this issue on the European policy agenda [5], is related to the identification and networking of reference centers, with the aim of improving care and patient's quality of life.

The RARECARENet and RITA2 project have proposed general criteria for identifying centers of reference for rare cancers. In Europe, several countries (Belgium, the Netherlands, Ireland, Germany, UK) have already 
Table 1 Minimum requirements needed to define a referral center for rare cancers

SICO survey items

Expertise, experience and activities

Volume of treated patients (to be defined by type of tumor)

Documented adherence to validated guidelines

Presence of mono or multi-center active trials and/or regularly published activity

Educational/training activities for experts and professionals in the area

Guidelines development

\section{Organization}

Documented and shared diagnostic/therapeutic pathways which also include relationships with local services/general practitioners/paediatricians

Formalized multidisciplinary teams

Transparent quality assurance systems

\section{Structure}

Accessibility to all surgical specialties including minimally invasive and/or reconstructive when indicated

Access to advanced diagnostic methods

Accessibility to preoperative RT treatments

Dedicated technology (IORT, HIFU, Hadrontherapy, HIPEC, ILP) "in house" or through collaborations with other centers

E-health platforms to process and share information, medical image or medical records supported by sufficient structural and technological human resources

Database for the prospective collection of clinical data

Tissue banking (in-house or virtual/networked with other centers)

identified reference centers for some rare cancers: France has an oncology project entirely dedicated to rare cancers, while the European Community (http://ec.europa.eu/ health/ern/policy/index_en.htm) is launching a call for the definition of the European Reference Networks (ERN), by promoting international collaboration between centers of reference from different countries.

In Italy, the Italian Society of Surgical Oncology (SICO) and the Italian Society of Surgery (SIC), in partnership with the main national oncological scientific societies, have conceived a project to contribute to the debate concerning the requirements and minimum standards for the definition of a reference center for rare cancers, providing homogeneous criteria which can standardize the process of identification of such centers throughout the national territory. This will be the basis to define the essential requirements for a network of excellences integrating the already active working groups, considering the crucial role of surgery in rare tumors.

The RARECARENet project data [2] has shown that $65 \%$ of all rare solid tumors receive a surgical treatment, compared to 35 and $28 \%$ referred to radiation therapy and chemotherapy. Considering the treatment of the rare solid tumors by stage, $82 \%$ of patients with a localized disease benefits of surgery.

With the aim of finding which criteria are needed to identify referral centers for rare cancers, the Italian Society of Surgical Oncology (SICO) administered to its members a survey investigating questions, derived from the RARECARENet experience, concerning the minimum requirements to define expertise, organization and structural peculiarities of a referral center for rare cancers (Table 1). Results were shared with the board of the Italian Society of Surgery, the Italian Association of Medical Oncology, the Italian Association of Pathology and the Italian Association of Radiation Oncology.

The criteria that emerged from the SICO survey are consistent with those proposed in Europe by RARECARENet project, such as:

- Assessment of expertise and experience based on observed and treated patients volume (to be defined by type of tumor).

- Quality of experience evaluated by scientific evidence and characterized by a multidisciplinary approach.

- Ability to define clinical care pathways at national and international level.

- Ability to develop clinical guidelines for the treatment of specific tumor types and for the governance and management of the service/network.

- Ability to organize events and training activities.

- Promotion and participation in clinical, translational and epidemiological research.

- Organization or endorsing of patient's empowerment activities.

- Availability of e-health platform to process and share information, medical imaging or medical records. 
- Adequate human, structural and technological resources.

- Continuous monitoring and evaluation of the quality of services.

These are general criteria which may answer to the general problems that arise from the management of rare cancers, but they may well be adapted to the definition of referral centers also for common cancers, a process which is presently ongoing in several European countries.

As rare tumors include different and heterogeneous hystotypes, it will be also important to define tumorspecific criteria, that is to adapt the aforementioned criteria at least to the 12 main rare cancers families (head and neck, rare thoracic tumors, digestive tract, male and female urogenital tract, endocrine glands, central nervous system, non-melanoma skin tumors, neuroendocrine tumors, sarcomas, certain haematological malignancies and childhood cancer).

In conclusion, the SICO survey underlined the need for specialist centers, in order to to optimise the treatment of rare tumours. Given their numerosity and complexity, nonspecialized pathologists might be lacking of experience, and such diagnostic uncertainties could result into inappropriate treatments and delays. Surgery for rare cancers may not necessarily be more demanding than the standard practice, from a technical point of view; however, the lack of oncological understanding of the disease may often lead to mismanagement, even in the absence of technical challenges. A multidisciplinary clinical approach at the very beginning of the patient's journey is highly advisable to allow the setting of a proper and tailored treatment plan.

The recognition or constitution of expert centers for rare cancers is the first step towards the establishment of a Reference Network. Cancer Networks have been viewed as a strategy to improve outcomes by centralizing patients and decreasing patients migration: networking between centers of excellence and surroundings hospitals is a key element to ensure that expertise travels, rather than patients. This model could well serve as the basis of a re-organization of cancer care in Italy also for common tumors, as referral centers are known to improve all cancer related outcomes, no matter what the frequency of the tumor is.

\section{Compliance with ethical standards}

Conflict of interest The authors declare that they have no conflict of interest.

Ethical approval All procedures performed in studies involving human participants were in accordance with the ethical standards of the institutional and/or national research committee and with the 1964 Helsinki declaration and its later amendments or comparable ethical standards.

Research involving human participants and/or animals This article does not contain any studies with human participants or animals performed by any of the authors.

Informed consent Although for this type of study formal consent is not required, informed consent was obtained from all individual participants included in the study.

\section{References}

1. AIRTUM Working Group. Italian cancer figures, report (2014) Prevalence and cure of cancer in Italy. Epidemiol Prev 38(6 Suppl 1): $1-122$

2. Mallone S, De Angelis R, van der Zwan JM, RARECARE WG et al (2013) Methodological aspects of estimating rare cancer prevalence in Europe: the experience of the RARECARE project. Cancer Epidemiol 37(6):850-856

3. Trama A, Mallone S, Ferretti S, RITA working group et al (2012) The burden of rare cancers in Italy: the surveillance of rare cancers in Italy (RITA) project. Tumori 98(5):550-558

4. Busco S, Buzzoni C, Mallone S, AIRTUM Working Group et al (2016) Italian cancer figures-Report 2015: The burden of rare cancers in Italy. Epidemiol Prev 40(1 Suppl 2):1-120

5. http://www.rarecancerseurope.org. Accessed May 2016 